\title{
Extensor Triggering of land: A Rare Presentation and its Management
}

\section{JER}

\author{
Schiller Jos, Abdul Gafoor PM, Mahesh B, Rahul Mohan, Sajesh BS \\ Department of Orthopaedics, Baby Memorial Hospital, Calicut, Kerala, India.
}

\section{Abstract:}

Trigger finger commonly refers to flexor tendon triggering which occurs due to pathologic changes in first annular pulley or the flexor tendons. Extensor triggering is a rare condition caused by disruption or laxity of the sagittal bands that stabilize the extensor digitorum communis tendon over the metacarpal head. Here we are reporting a rare case of a 14 year old girl who presented with four year history of acquired idiopathic extensor triggering of multiple fingers of both hands. Physiotherapy and other modalities of conservative management failed to prevent triggering, hence surgical correction by the Carroll tendon slip procedure was performed to centralize the extensor tendon. She underwent post-operative rehabilitation and three year follow up showed excellent response to the treatment.

Key words: Hand, Metacarpal Bones, Physical Therapy Modalities, Tendons, Trigger Finger Disorder.

\section{Introduction}

Trigger finger commonly refers to flexor tendon triggering which is clinically diagnosed by a sudden extension of the affected finger at the proximal interphalangeal joint (PIPJ) $[1,2]$. It happens when a flexor tendon passes through the first annular (A1) pulley with palpable triggering over the palmar side of the metacarpophlangeal joint (MCPJ) due to pathologic changes within the $A 1$ pulley or the flexor tendons $[1,2]$.

Subluxation of the extensor digitorum communis (EDC) per se is an uncommon cause for trigger finger [3]. On extensive search, we found very few reports of extensor tendon triggering in the literature [4-7]. Extensor triggering is usually caused by disruption or laxity of the sagittal bands that stabilize the extensor digitorum communis tendon over the metacarpal head $[3,8]$. It is also known as boxers knuckle as it is commonly found in pugilists [3]. The middle finger is the commonest site followed by the little finger, and the radial sagittal band is more commonly affected than the ulnar bands [8]. Even though trauma is the commonest cause, inflammatory joint disease like rheumatoid arthritis can also contribute [3]. Congenital absence of the sagittal bands has also been reported [9].

Here we are presenting a rare case of acquired extensor triggering of multiple fingers of both the hands which was neither traumatic nor associated with any inflammatory disease and it's excellent functional outcome with surgical management.

\section{Corresponding Author: Dr. Abdul Gafoor PM}

Email: abdulgafoorpm@gmail.com

Received: March 25, 2016 | Accepted: July 3, 2016 | Published Online: July 5, 2016

This is an Open Access article distributed under the terms of the Creative Commons Attribution License (creativecommons.org/licenses/by/3.0)

Conflict of interest: None declared | Source of funding: Nil | DOl: http://dx.doi.org/10.17659/01.2016.0073 


\section{Case Report}

A 14 year old, right handed girl presented with a four year history of painful triggering of index, middle and ring finger of both hands, right side more symptomatic than the left. Before presenting to our hospital she had undergone treatment elsewhere initially conservative and later surgical release of A1 pulleys of all the affected fingers after being misdiagnosed as flexor tendon triggering of multiple fingers. However her symptoms persisted and got aggravated with time.

On physical examination there was a visible as well as palpable "click" on the dorsum of the hand over the metacarpal head with radial subluxation of EDC with flexion of the fingers. On attempting extension there was triggering with a snap associated with reduction of EDC. The patient had generalized ligamentous laxity and bilateral peroneal tendon subluxation [Fig. 1,2].

As the symptoms were persisting and not responding to conservative management, it was decided to perform an extensor tendon centralization procedure, on the affected fingers of the right hand which was more symptomatic. Carroll tendon slip procedure was done to centralize the extensor digitorum communis (EDC) tendon. In this technique distally based slip of EDC tendon on ulnar side was prepared and routed deep to affected tendon and around radial collateral ligament which was deficient. It was then sutured to itself after tensioning to centralize tendon [Fig.3-5].

Volar splint in functional position of the hand was used for 2 weeks after the surgery followed by 3 months of physiotherapy which included 1 month each of passive mobilization, active mobilization and resisted exercises. Patient improved symptomatically and regained full function and strength. She could resume her activities of daily living. Three year follow up showed excellent functional outcome [Fig.6].
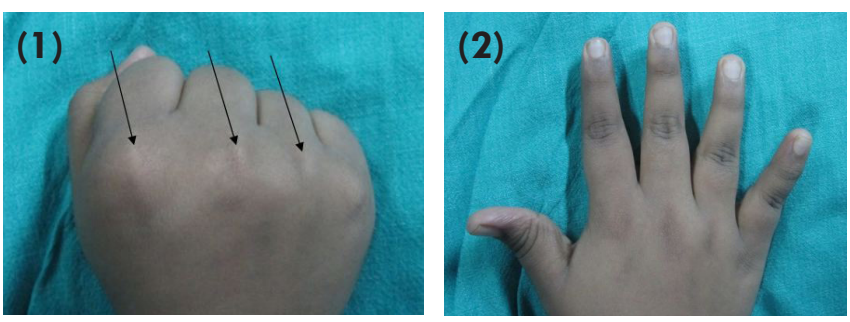

Fig.1,2: Subluxation of EDC with flexion and reduction with triggering on extension.

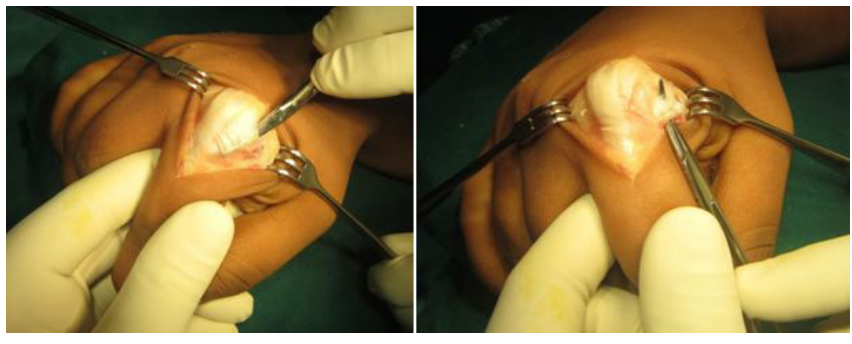

Fig.3: The radial sagittal band was found to be lax preoperatively.

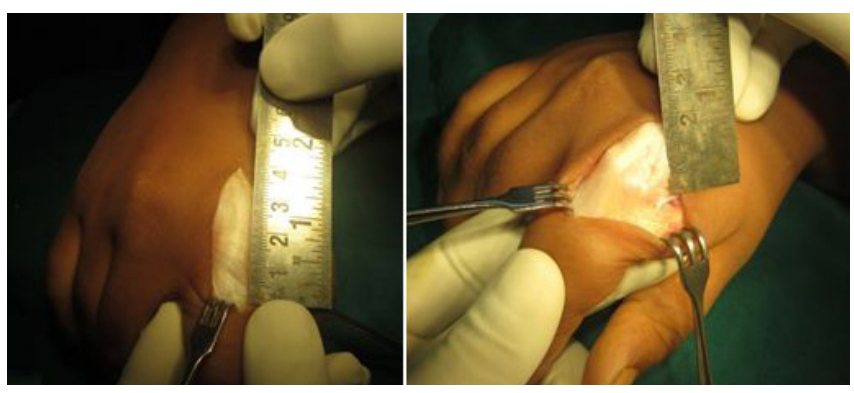

Fig.4: The required length was measured and distally based ulnar side slip taken.

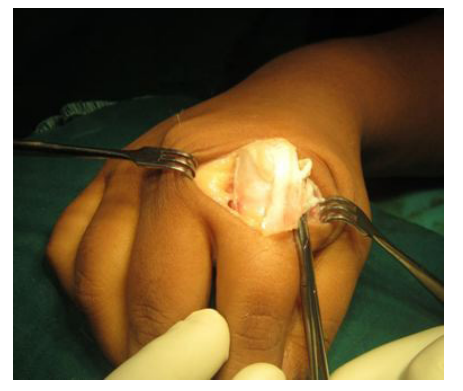

Fig.5: Slip sutured to itself after tensioning to centralize the tendon. 


\section{Discussion}

Flexor tendon triggering is due to stenosing tenosynovitis of the flexor tendons, which is actually caused by a mismatch between the volume of the flexor tendon sheath and its contents $[1,2]$. The diagnosis and treatment of flexor tendon triggering is quite familiar to most orthopedic surgeons however, extensor triggering is an uncommon condition that may be missed unless looked for carefully [10]. It may be misdiagnosed for a flexor triggering due to the snapping sensation that occurs when the extensor tendon shifts back and forth between the ulnar groove and its normal position over the metacarpal head [3].

The extensor tendon at the dorsum of the hand is stabilized by a complex retinacular system called the dorsal hood [11]. The dorsal hood is made up of 3 structures: the sagittal, oblique, and the transverse bands $[11,12]$. At the MCP level the extensor tendons are stabilized by the sagittal bands and more distally between the MCP and the proximal IP joints by the transverse and the oblique bands, corresponding to a fibrous extension of the interosseous and lumbrical muscles, respectively $[11,12]$. The sagittal band is divided into a thin superficial layer and a thicker deep layer forming a channel through which the extensor tendon runs [11]. The most important structure of the dorsal hood providing stability of the extensor tendon at the MCP level are the sagittal bands. The tendons tend to follow the ulnar slope of the metacarpal head and generally would dislocate in an ulnar direction on flexion [13]. The contour of the MCP joint articular surface induces an ulnar deviation of the fingers $[13,14]$. The radial sagittal band is usually thinner and longer than the ulnar component, making ulnar instability of the involved extensor tendon more common [12-14].

Kardashian et al. [5] reported two cases of EPL stenosing tenosynovitis with triggering of

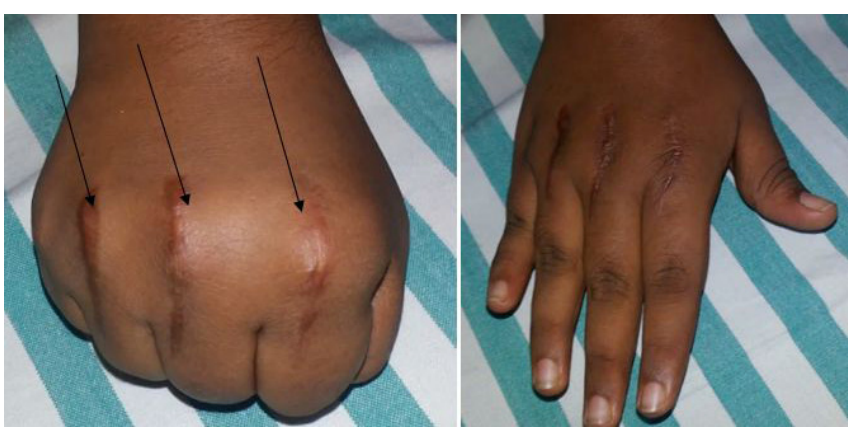

Fig.6: Three months post-surgery: Extensor tendon stable over the metacarpal head, patient regains full functional status.

the thumb in the area of Lister's tubercle. Durand et al. [7] reported triggering in the lateral slip of the extensor mechanism on a Bouchard's node. Some authors have described extensor triggering by impingement of tendons on the extensor retinaculum [15]. Low energy or atraumatic sagittal band ruptures or dysfunctions are reported rarely in the literature [12]. Rayan and Murray classified closed sagittal band injuries. Type I is the sagittal band injury without extensor tendon instability; type II is the injury with tendon subluxation; and type III, injury with tendon dislocation [8].

Conservative managements like extension splints are useful in early presenting acute injuries. Surgical management is usually needed if conservative treatment fails or in chronic cases which includes direct tendon repair and tendon centralization procedures $[8,12]$. Our case was chronic and atraumatic, so we opted for a tendon centralizing procedure. Various centralization procedures have been described for chronic subluxation and its selection depends on the pathoanatomy of the injury and experience of the surgeon [8].

\section{Conclusion}

Extensor triggering is a rare condition, but proper knowledge and through clinical examination can help 
in the diagnosis. Selection of appropriate treatment method and a good post-operative rehabilitation protocol can assure an excellent functional result as in our case.

\section{References}

1. Tarbhai K, Hannah S, von Schroeder HP. Trigger finger treatment: a comparison of 2 splint designs. J Hand Surg Am. 2012;37(2):243249.

2. Cakmak F, Wolf MB, Bruckner T, Hahn P, Unglaub F. Follow-up investigation of open trigger digit release. Arch Orthop Trauma Surg. $2012 ; 132(5): 685-691$.

3. Chillag SA, Greenberg S. Images in clinical medicine. An unusual cause of trigger finger. $\mathrm{N}$ Engl J Med. $2011 ; 365(7):$ e 14.

4. Alberton GM, High WA, Shin AY, Bishop AT. Extensor triggering in de Quervain's stenosing tenosynovitis. J Hand Surg Am. $1999 ; 24(6): 1311-1314$.

5. Kardashian G, Vara AD, Miller SJ, Miki RA, Jose J. Stenosing synovitis of the extensor pollicis longus tendon. J Hand Surg Am. $2011 ; 36(6)$ : 1035-1038.

6. O'Rourke PJ, O'Sullivan T, Stephens M. Extensor tendon sheath stenosis resulting in triggering of the little finger. J Hand Surg Br. 1994; 19(5):662663.

7. Durand S, Gaujoux G, Macquillan A. Triggering of the lateral slip of the extensor mechanism on a Bouchard's node. J Hand Surg (Eur). $2011 ; 36(4): 340-341$.

8. Rayan GM, Murray D. Classification and treatment of closed sagittal band injuries. J Hand Surg Am. 1994;19(4):590-594.

9. Posner MA, McMahon MS. Congenital radial sublaxation of the extensor tendons over the metacarpophalangeal joints: a case report. J Hand Surg. 1994;19(4):659-662.

10. Park SE, Kim YY, Ji JH, Lee HH, Jeong JJ. Double triggering of extensor digiti minimi: a case report. Arch Orthop Trauma Surg. 2013;133(3):429-432.

11. Kichouh M, Vanhoenacker F, Jager T, Van Roy $P$, Pouders $C$, Marcelis S, et al. Functional anatomy of the dorsal hood or the hand: correlation of ultrasound and MR findings with cadaveric dissection. Eur Radiol. 2009;19(8):1849-1856.

12. Capo JT, Shamian B, Rossy W, Hashem J. Closed sagittal band injury due to low energy trauma. Am J Orthop (Belle Mead NJ). 201 2;41 (8):374377.

13. Hakstian RW, Tubiana R. Ulnar deviation of the fingers. The role of joint structure and function. J Bone Joint Surg Am. 1967;49(2):299-316.

14. Tubiana $R$, Valentin P. The anatomy of the extensor apparatus of the fingers. Surg Clin North Am. 1964;44:897-906.

15. Khazzam M, Patillo D, Gainor BJ. Extensor tendon triggering by impingement on the extensor retinaculum: a report of 5 cases. J Hand Surg Am. 2008;33(8):1397-1400. 